\title{
Research of the blockage ratio on the aerodynamic performances of high speed elevator
}

\author{
Xuebin Wang ${ }^{1, \text { a }}$, Zheng Lin ${ }^{1}$, Ping Tang ${ }^{1}$, Zhangwei Ling ${ }^{1}$
}

${ }^{1}$ Zhejiang Provincial Special Equipment Inspection and Research Institute, Kai xuan Road 211, Hangzhou, 310020, P. R. China

a wxbhzzj@sina.com

Keywords: High speed evaluator; Aerodynamic performance; Blockage ratio; CFD

\begin{abstract}
The three-dimensional turbulent flow of high speed elevator is numerical simulated using the static uncompressible Navier-Stokes equations. The equations are solved using finite volume method with second order upwind spatial discretization. The car of the high speed elevator is a box with the length of $2.3 \mathrm{~m}$, with the width of $1.7 \mathrm{~m}$, and height of $2.4 \mathrm{~m}$. Blockage ratios of $0.595,0.512$ and 0.446 are researched on the effects of the aerodynamic performances. A serial of elevator well is built according to the blockage ratio of $0.595,0.512$ and 0.446 . The aerodynamic performance especially the drags of the elevator cars are computed. The results show that with the blocking ratio decreases, the pressure and the drag forces of the high-speed elevator decrease.
\end{abstract}

\section{Introduction}

With the rapid development of China's economy, the number and the height of tall buildings increase rapidly. It is predicted that there will be 164 tall buildings with the height greater than $250 \mathrm{~m}$ in China before 2018. In addition to the tall building number increasing, its height in recent years has been refreshed. The number of the high-rise buildings whose heights are greater than $500 \mathrm{~m}$ also increases rapidly, such Shanghai Tower [1]. Thus the market demand of the high-speed elevator (more than $12 \mathrm{~m} / \mathrm{min}$ ) has been increased significantly in China. However, with the increase of the elevator speed, the influences of elevator well and elevator car on aerodynamic characteristics are not negligible. Especially in the region between elevator well and elevator car, the sudden decrease of the flow area results in the dramatically increase of air speed. The quality of the flow in the region influences the stability, security, and noise of a high-speed elevator [2, 3]. Therefore it is necessary to study the effects of high-speed elevator blocking ratio on the aerodynamic performances.

The research methods on aerodynamic performances include experiments method and numerical method [4,5]. With the maturing of CFD method in recent years, the numerical method is being widely used in the elevator flow simulations. Yang have simulated two dimensional flow and computed the aerodynamic performance using the CFD method[6]. Hisashi [7] used CFD method to simulated three-dimensional flow, his results show that the flow conditions affect the elevator car's aerodynamic performances and the turbulent flow is a main source of the aerodynamic noise. The flow is numerical simulated and aerodynamic forces are computed using the Fluent software. The influences of the blockage ratio on the aerodynamic forces are further considered.

\section{The evaluator model}

The car of the high speed elevator studied in this paper is a box with the length of $2.3 \mathrm{~m}$, with the width of $1.7 \mathrm{~m}$, and height of $2.4 \mathrm{~m}$. The diversion covers are built with conic surface with the height of $0.8 \mathrm{~m}$. The height of the elevator well is $100 \mathrm{~m}$, while the widths and the lengths are $2.4 \times 2.8 \mathrm{~m}$ 、 $2.6 \times 3.0 \mathrm{~m}$ and $2.8 \times 3.2 \mathrm{~m}$, respectively. The corresponding blockage ratios are $0.595,0.512$ and 0.446, respectively. To reduce the computational cost, only quarters of the models are built, parts of them are shown in Fig1. 

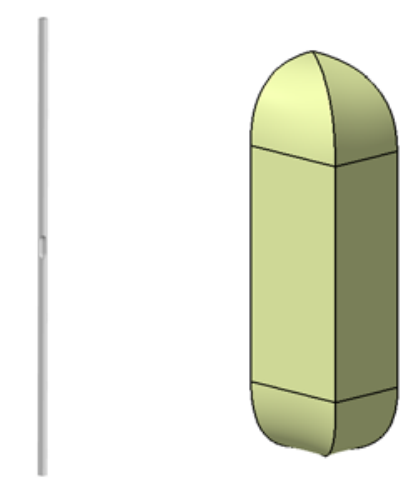
a) Flow region
b) the elevator car model

Fig.1. The elevator system model

During the grid generations of the elevator using Ansys.Icem software, the hybrid grid technology is used to ensure the quality of the grid at the same time reduce the number of cells. Fig 2 (a) and (b) show the local grid capsules near the car, the grids are generated with tetrahedron elements while hexahedron elements are used in the wall boundary regions. In the far field, the structure grid technology is adopted. The meshes of the symmetry plane of different blockage ratio are shown in Fig.3.

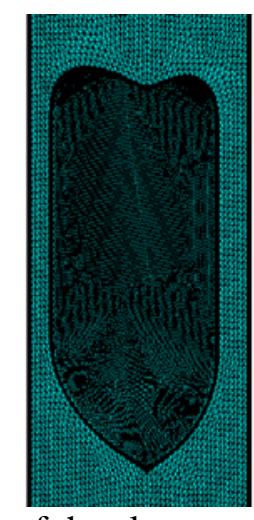

b) mesh of the elevator car

Fig.2. High speed evaluator models with different diversion cover

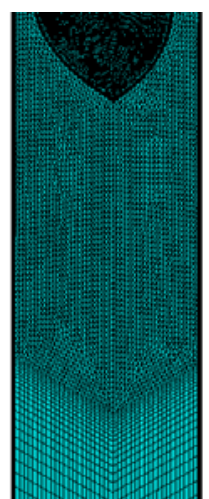

c) mesh of the symmetry plane

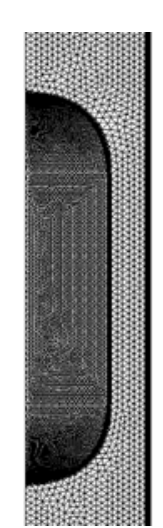

b) ratio of 0.51

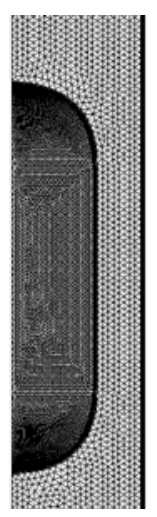

c) ratio of 0.446

a) ratio of 0.595

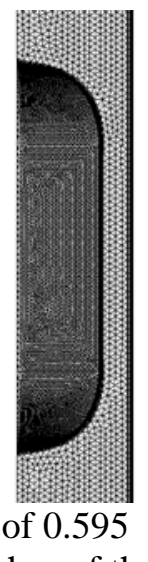

Fig.3. meshes of the symetry plane with different blockage ratio

\section{Computation method}

In the Cartesian coordinate system, the three-dimensional steady N-S equations in a conservative differential form on a moving mesh can be written as

$$
\frac{\partial}{\partial t} \int_{\Omega} \vec{W} d \Omega+\oint_{\partial \Omega}\left(\vec{F}_{c}-\vec{F}_{v}\right) d S=0
$$

Where $t$ is time, $\Omega$ is the control volume and $\partial \Omega$ is the volume surface, $\vec{W}$ is the conserved 
variables, $\vec{F}_{c}$ and $\vec{F}_{v}$ are convective part and viscosity part. The finite element method is used to solve the equations with $k-\omega$ turbulence model. A second order cell-centered scheme is use.

The boundary conditions are: the entrance of the elevator well is the inlet with velocity of $12 \mathrm{~m} / \mathrm{s}$; the exit is the pressure outlet; the elevator car is station wall; the elevator well is moving wall with velocity of $12 \mathrm{~m} / \mathrm{s}$; Symmetric boundary conditions are used at the symmetric planes.

\section{Results and discussion}

Figure 3 shows the variation of the high speed elevator drag force with different blocking ratio. It is shown that with the increase of the blockage ratio, the drag force increase gradually nonlinearly.

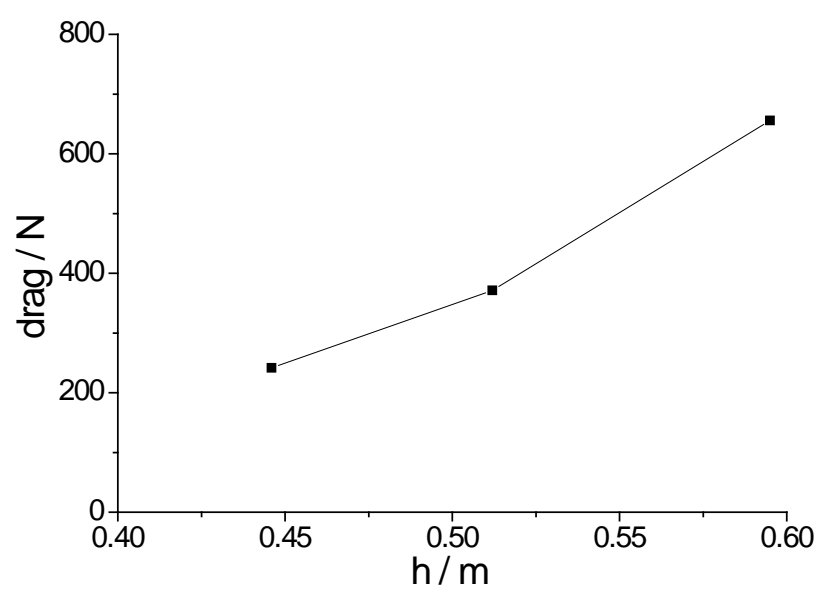

Fig.4. Drags of the elevator cars

The pressures contours of the high speed elevator car are shown in Fig 5. It is shown that the pressure of elevator with the ratio of 0.595 is $200 \mathrm{~Pa}$ and the high pressure region is quite large. With the blockage ratio of elevator decreases, both the drag forces and the high pressure region of the elevator decrease.

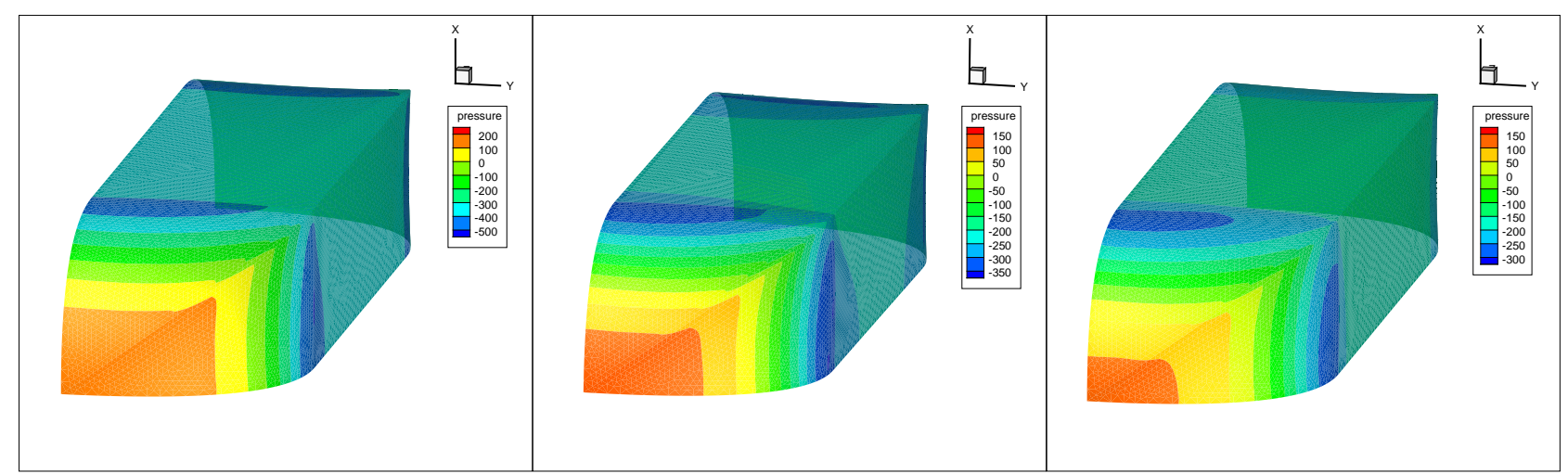

a) ratio $=0.595$

b) ratio $=0.51$

c) ratio $=0.51$

Fig.5. Pressures of the elevator car with different blockage ratios

The velocity contours of the symmetry plane are shown in Fig 6. With the blockage ratio of elevator decreases, the velocity magnitude decrease. The velocity contours of the flow between elevator car and the elevator well has a more homogeneous distribution, and the wake region of the car shrinks. 


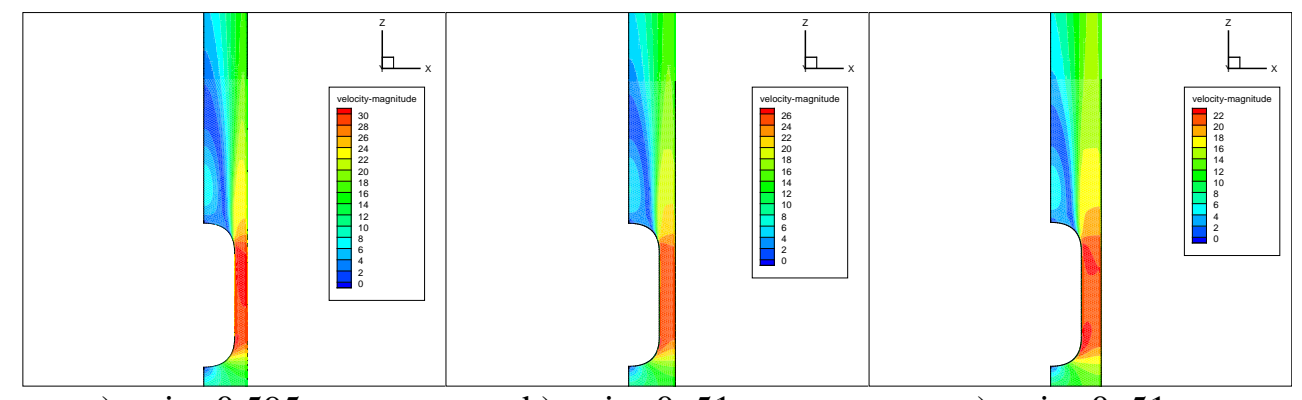

a) ratio $=0.595$

b) ratio $=0.51$

c) ratio $=0.51$

Fig.6. velocity of the symetry plane with different blockage ratios

A X-Y cut plane at the middle of car is made, and The velocity contours of the cut plane are shown in Fig 7. With the blockage ratio of elevator decreases, the velocity magnitude of the cut plane decreases from $30 \mathrm{~m} / \mathrm{s}$ to $22 \mathrm{~m} / \mathrm{s}$. Also, the velocity contours between elevator car and the elevator well has a more homogeneous distribution.

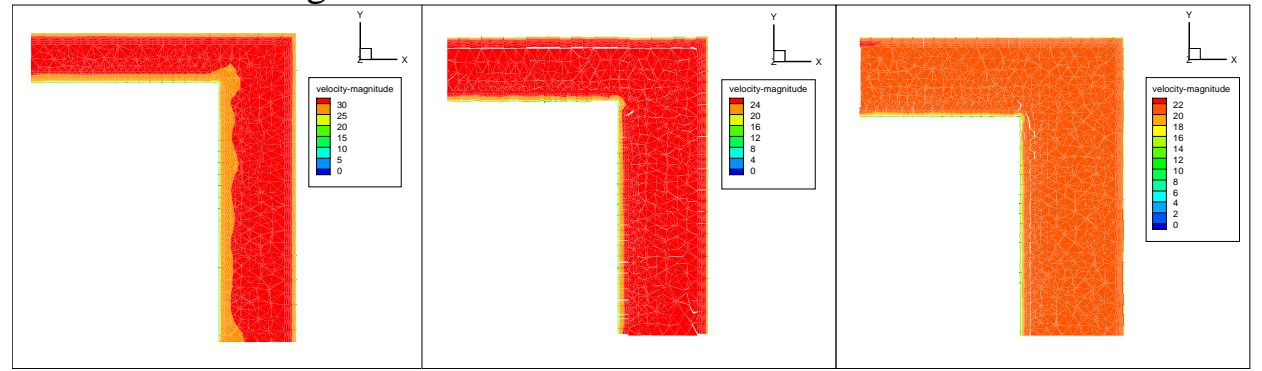
a) ratio $=0.595$
b) ratio $=0.51$
c) ratio $=0.51$

Fig.7. velocity of the cut plane with different blockage ratios

\section{Conclusion}

The flows of the high speed elevator are simulated using the CFD method and the influences of the blockage ratio on the aerodynamic performances are studied in this paper. With the blockage ratio of elevator decreases, the drag forces and pressures of the elevator decrease, the velocities of the flow region decrease and the velocity of the flow between elevator car and the elevator well has a more homogeneous distribution.

\section{Acknowledgment}

This project is supported by the Major Project of Zhejiang Bureau of Quality and Technical Supervision (NO. 20130128 ).

\section{References}

[1]. Jian Jiang, Lingzhu Chen, Shouchao Jiang, Guo-Qiang Li, Asif Usmani. Fire safety assessment of super tall buildings: A case study on Shanghai Tower[J]. Case Studies In Fire Safety 4 (2015), 28-38.

[2]. John H. Klote and George T. Tamura. Elevator piston effect and the smoke problem[J]. Fire Safety Journal, 1986, 11 ( 2 ) :227 233.

[3]. Shigate M. Inaba H. Development of Super High Speed Elevators[J]. Elevator Word. 1995, (4): 69 73

[4] DUAN Ying, SHEN Guo-xing. Aerodynamic testing simulation facility for high speed elevator[J]. Journal of Beijing University of Aeronautics and Astronautics, 2004, 30(5): 444-447.

[5] TORSTEN Wintergerste, SABINE Sulzer. Low noise, high-speed elevator[J]. Sulzer Technical Review, 2004, (3): 11-13. 
[6] Yang H.W, So A.T.P. A 2-Dimensional Aerodynamic Model for Super high speed Elevators[J]. International Journal of Elevator Engineering. 1998, (2): 19 32

[7] HISASHI Matsuda, YOSHITAKA Fukuyama and YASUYUKI Yokono. Flow around an elevator model (the effect of Apron on flow around a car during downward motion) [C]. The 69th National Conference of Japanese Mechanism Academy. Nagoya, Japan, 1991, 540-542. 